\title{
FACTORALITY IN QUOTIENTS OF LINEAR $\boldsymbol{G}_{m}$-ACTIONS
}

ANDY R. MAGID

Let $k$ be an algebraically closed field of characteristic 0 and let $G_{m}=G L(1, k)$ be the multiplicative group of $k$ regarded as an algebraic group. Suppose $G_{m}$ acts rationally on the $n$-dimensional $k$ vector space $V$. The purpose of this paper is to examine factorality in the ring $R=k[V]^{G_{m}}$ of $G_{m}$-invariant polynomial functions on $V$; that is, to compute the divisor class group $C \ell(R)$.

We can always choose a basis $x_{1}, \cdots, x_{n}$ of $V$ such that $G_{m}$ acts diagonally with respect to this basis, i.e. $t$ in $G_{m}$ sends $x_{i}$ to $t^{\lambda_{i}} x_{i}$. For example, if $n=2, \lambda_{1}=1$, and $\lambda_{2}=-1$, then $R=k\left[x_{1} x_{2}\right]$ and $C \ell(R)=1$. If $n=3, \lambda_{1}=1, \lambda_{2}=2$, and $\lambda_{3}=-4$, then $R=$ $k\left[x_{1}{ }^{4} x_{3}, x_{2}{ }^{2} x_{3}, x_{1}{ }^{2} x_{2} x_{3}\right]$, so in terms of generators and relations, $R$ is generated by $a=x_{1}{ }^{4} x_{3}, b=x_{2}{ }^{2} x_{3}, c=x_{1}{ }^{2} x_{2} x_{3}$ subject to the single relation $c^{2}=a b$. Then $C l(R)=\mathrm{Z} / 2 \mathrm{Z}$. If $n=4, \lambda_{1}=\lambda_{2}=1$ and $\lambda_{3}=\lambda_{4}=-1$, then $R=k\left[x_{1} x_{3}, x_{2} x_{3}, x_{1} x_{4}, x_{2} x_{4}\right]$ so $R$ is generated by $a=x_{1} x_{3}, b=x_{2} x_{3}, c=c_{1} x_{4}, d=x_{2} x_{4}$, subject to the single relation $a d=b c$. Then $C \ell(R)=\mathrm{Z}$. Thus $C \ell(R)$, in these examples, is cyclic, but it can be infinite cyclic, finite cyclic, or trivial.

The general result established here is the following: suppose all $\lambda_{i}$ are non-zero with exactly $p$ positive. If $p$ and $n-p$ are larger than $1, C \ell(R)=Z$. If $p=1$ or $n-p=1, C \ell(R)$ is finite cyclic of computable order - the computation depends on some number-theoretic calculations modulo $\lambda_{n}$ (if $n-p=1$ ) or $\lambda_{1}$ (if $p=1$ ). The approach to this determination is via a study of the geometric quotient prevariety $W=(V-0) / G_{m}$, whose existence we establish, and we show that $\operatorname{Pic}(W)=Z$, always.

For convenience, we establish some notational conventions in the beginning of the paper and conserve these throughout for proofs, although the theorems are stated without the conventions.

We assume throughout that $k$ is an algebraically closed field of characteristic zero, and all our pre-varieties are over $k$. We identify pre-varieties with their $k$-closed points. We use $k[W]$ to stand for $\Gamma\left(W, O_{W}\right)$ if $W$ is a pre-variety and ()$^{*}$ to denote the units functor. We also use the relative units functor $U_{k}$, whose value on the pre-variety $W$ is $k[W] * / k^{*}$.

Received by the editors on December 26, 1975, and in revised form on July 5 , 1976. 
Definition 1. Let $n, m, \lambda_{1}, \cdots, \lambda_{n+m}$ be positive integers. Then $\left[(n, m), \lambda_{1}, \cdots, \lambda_{n+m}\right]$ denotes $k^{(n+m-1)}-0$ with coordinates $x_{1}, \cdots$, $x_{n+m}$ and $G_{m}$ action given by $x_{i} \rightarrow t^{\lambda_{i}} x_{i}, 1 \leqq i \leqq n$, and $x_{i} \rightarrow t^{-\lambda_{i}} x_{i}$, $n+1 \leqq i \leqq n+m$. The action is called reduced if the greatest common divisor of $\lambda_{1}, \cdots, \lambda_{n+m}$ is 1 .

Remark. Let $d=$ g.c.d. $\left(\lambda_{1}, \cdots, \lambda_{n+m}\right)$ and let $\lambda_{i}{ }^{\prime}=\lambda_{i} / d$. Suppose $Z$ is a geometric quotient of $\left[(n, m), \lambda_{1}{ }^{\prime}, \cdots, \lambda_{n+m}{ }^{\prime}\right]$ by $G_{m}$. Then the quotient $\left[(n, m), \lambda_{1}, \cdots, \lambda_{n+m}\right] / G_{m}$ exists and equals Z: for let $f: G_{m}$ $\rightarrow G_{m}$ be the $d$ th power map. Then $G_{m}$ acting on $\left[(n, m), \lambda_{1}{ }^{\prime}, \cdots\right.$, $\left.\lambda_{n+m}^{\prime}\right]$ via $f$ is equivalent to $G_{m}$ acting on $\left[(n, m), \lambda_{1}, \cdots, \lambda_{n+m}\right]$. So whenever it is convenient we may take the action to be reduced.

Fix $(n, m), \lambda_{1}, \cdots, \lambda_{n+m}$, let $V=\left[(n, m), \lambda_{1}, \cdots, \lambda_{n+m}\right]$ and let $V^{\prime}=[(n, m), 1, \cdots, 1]$. Let $\phi: V^{\prime} \rightarrow V$ send $x_{i}$ to $x_{i}^{\lambda_{i}}$. It is trivial to verify that $\phi$ is a surjective $G_{m}$-equivariant morphism. For any integer $\ell$, let $\Gamma(\ell)$ denote the group of $\ell$ th roots of unity in $k$. Let $G=\Gamma\left(\lambda_{1}\right)$ $\times \cdots \times \Gamma\left(\lambda_{n+m}\right)$. Then $g=\left(a_{1}, \cdots, a_{n+m}\right)$ in $G$ acts on $V^{\prime}$ by $g\left(x_{1}, \cdots\right.$, $\left.x_{n+m}\right)=\left(\alpha_{1} x_{1}, \cdots, \alpha_{n+m} x_{n+m}\right)$. The actions of $G$ and $G_{m}$ on $V^{\prime}$ commute and $\phi$ induces an isomorphism of $V^{\prime} / G$ with $V$. For each $i=1, \cdots$, $n+m$, let

$$
V_{i}^{\prime}=V^{\prime}-\left(x_{i}=0\right) \text { and } V_{i}=V-\left(x_{i}=0\right) .
$$

The $V_{i}{ }^{\prime}$ are open and $G_{m}$-stable in $V^{\prime}$, and their union covers $V^{\prime}$. Similar remarks apply to the $V_{i}$. Also, $\phi\left(V_{i}{ }^{\prime}\right)=V_{i}$.

For each $i$, let $\left.W_{i}{ }^{\prime}=k^{(n+m-1}\right)$ with coordinates $x_{1}, \cdots, \hat{x}_{i}, \cdots, x_{n+m}$, where $\hat{x}_{i}$ means $x_{i}$ is deleted. For each $i$ there are $G_{m}$-equivariant isomorphisms $\sigma_{i}: G_{m} \times W_{i}{ }^{\prime} \rightarrow V^{\prime}$ defined as follows:

$$
\begin{aligned}
\text { For } i \leqq & n, \sigma_{i}\left(t, x_{1}, \cdots, \hat{x}_{i}, \cdots, x_{n+m}\right) \\
= & \left(t x_{1}, \cdots, t x_{i-1}, t, t x_{i+1}, \cdots, t x_{n}, t^{-1} x_{n+1}, \cdots, t^{-1} x_{n+m}\right), \\
& \sigma_{i}^{-1}\left(x_{1}, \cdots, x_{n+m}\right) \\
= & \left(x_{i}, x_{i}^{-1} x_{1}, \cdots, x_{i}^{-1} x_{i}, \cdots, x_{i}^{-1} x_{n}, x_{i} x_{n+1}, \cdots, x_{i} x_{n+m}\right) .
\end{aligned}
$$

For $i>n, \sigma_{i}\left(t, x_{1}, \cdots, \hat{x}_{i}, \cdots, x_{n+m}\right)$

$$
\begin{aligned}
= & \left(t x_{1}, \cdots, t x_{n}, t^{-1} x_{n+1}, \cdots, t^{-1} x_{i-1}, t^{-1} x_{i+1}, \cdots, t^{-1} x_{n+m}\right), \\
& \sigma_{i}{ }^{-1}\left(x_{1}, \cdots, x_{n+m}\right) \\
= & \left(x_{i}{ }^{-1}, x_{i} x_{1}, \cdots, x_{i} x_{n}, x_{i}{ }^{-1} x_{n+1}, \cdots, x_{i}{ }^{-1} x_{i}, \cdots, x_{i}{ }^{-1} x_{n+m}\right) .
\end{aligned}
$$

For each $i, \sigma_{i}$ induces an isomorphism of $W_{i}{ }^{\prime}$ with $V_{i}{ }^{\prime} / G_{m}$. By uniqueness of geometric quotients, this means that the quotient $V^{\prime} / G_{m}$ exists 
and is covered by open sets isomorphic to $W_{i}{ }^{\prime}$. We denote these open sets in the quotient by $W_{i}{ }^{\prime}$ also.

Because the actions of $G$ and $G_{m}$ commute on $V^{\prime}, G$ acts on $V^{\prime} / G_{m}$, and each $W_{i}{ }^{\prime}$ is $G$-stable. Thus all $G$-conjugates of an element of $V^{\prime} / G_{m}$ lie in an open affine, and the quotient $V^{\prime} / G_{m} / G$ exists and is covered by the open affines $W_{i}=W_{i}{ }^{\prime} / G$. But $W_{i}{ }^{\prime} / G=V_{i}{ }^{\prime} / G_{m} / G=V_{i}{ }^{\prime} / G / G_{m}=$ $V_{i} / G_{m}$, and hence $V^{\prime} / G_{m} / G$ is a quotient of $V$ by $G_{m}$. (The above construction is a special case of the technique of $[4,6.1$, p. 543].) We have now shown:

THEOREM 2. The geometric quotient $\left[(n, m), \lambda_{1}, \cdots, \lambda_{n+m}\right] / G_{m}$ exists.

The geometry of the quotient may be quite complicated. We make a few remarks regarding it. Let $S=\{(i, j) \mid 1 \leqq i \leqq n, 1 \leqq j \leqq m\}$, let $V_{0}{ }^{\prime}=U\left(V_{i}{ }^{\prime} \cap V_{j+n}^{\prime}\right)$ (union over $(i, j)$ in $S$ ) and let $V_{0}=U\left(V_{i} \cap\right.$ $\left.V_{j+n}\right)$. Then $V_{0}{ }^{\prime}$ and $V_{0}$ are open $G_{m}$-stable subsets of $V^{\prime}$ and $V$, respectively. Let $C_{0}{ }^{\prime}=V_{0}{ }^{\prime} / G_{m}$ and $C_{0}=V_{0} / G_{m}$. Clearly $C_{0}=C_{0}{ }^{\prime} / G$. To determine $C_{0}{ }^{\prime}$, we consider the subset $C^{\prime}$ of $k^{(n m)}$ with coordinates $t_{i j}$ which is the locus of $t_{i j} t_{k \ell}-t_{i \ell} t_{k j}$ for all pairs $(i, j)$ and $(k, \ell)$ in $S$. It is easy to see that the morphism $V_{0}{ }^{\prime} \rightarrow C^{\prime}$ by $t_{i j}=x_{i} x_{j+n}$ defines an isomorphism of $C_{0}{ }^{\prime}$ with $C^{\prime}-0$, and thus $C_{0}{ }^{\prime}$ is an open subset of the affine variety $C^{\prime}$. $C^{\prime}$ is usually called the Segre cone of signature $(n, m)$, because $C^{\prime}$ is the cone in $k^{(n m)}$ lying over the image $P^{(n-1)} \times$ $\boldsymbol{P}^{(m-1)}$ in $\boldsymbol{P}^{(n m-1)}$ under the Segre embedding. The action of $G$ extends to $C^{\prime}$, and if $C=C^{\prime} / G, C_{0}$ is isomorphic to an open subset of $C$ whose complement is a single point (the image in $C$ of 0 in $C^{\prime}$ ).

We now describe the complements of $C_{0}{ }^{\prime}$ and $C_{0}$. First $V^{\prime}-V_{0}{ }^{\prime}$ $=Z_{1}{ }^{\prime} \cup Z_{2}{ }^{\prime}$, where $Z_{1}{ }^{\prime}=\left(k^{(n)}-0\right) \times 0$ and $Z_{2}{ }^{\prime}=0 \times\left(k^{(m)}-0\right)$, and $V-V_{0}=Z_{1} \cup Z_{2}$ where $Z_{i}=\phi\left(Z_{i}{ }^{\prime}\right)$. Now $Z_{i}{ }^{\prime}$ is $G_{m}$-stable, and $Z_{1}{ }^{\prime} / G_{m}=P^{(n-1)}$ and $Z_{2}{ }^{\prime} / G_{m}=P^{(m-1)}$, so $V^{\prime} / G_{m}=C_{0}{ }^{\prime} \cup P^{(n-1)} \cup$ $P^{(m-1)}$, and $V / G_{m}=C_{0} \cup P^{(n-1)} / G \cup P^{(m-1)} / G$, so the complements of $C_{0}{ }^{\prime}$ and $C_{0}$ are complete and, if $n>1$ or $m>1$, not affine. (If $n=m$ $=1$, it is easy to see that $V^{\prime} / G_{m}$ is the affine line with the origin doubled so $V^{\prime} / G_{m}$ is not separated).

Finally, we note that $V^{\prime} / G_{m}$ is non-singular since it is covered by the non-singular open sets $W_{i}{ }^{\prime}$ and that $V / G_{m}$ is normal since it is covered by the open sets $W_{i}=W_{i}{ }^{\prime} / G$.

We now begin the calculation of the Picard groups of the quotients.

Proposition 3. Pic $\left([(n, m), 1, \cdots, 1] / G_{m}\right)=\mathbf{Z}$.

Proof. In the established notation, $V^{\prime} \rightarrow V^{\prime} / G_{m}$ is a locally trivial fibration with fibre $G_{m}$ : the inverse image of $W_{i}{ }^{\prime}$ being $G_{m} \times W_{i}{ }^{\prime}$. We 
employ the exact sequence of [2, Theorem 5], noting that the demonstration in the reference does not require separation of the base. Since $U_{k}\left(V^{\prime}\right)=1$ and $\operatorname{Pic}\left(V^{\prime}\right)=1$, the exact sequence gives $1 \rightarrow U_{k}\left(G_{m}\right)$ $\rightarrow \operatorname{Pic}\left(V^{\prime} / G_{m}\right) \rightarrow 1$ exact, and hence the result.

To calculate Pic $\left(V / G_{m}\right)$, we use the fact [2, Lemma 2] that Pic (Y) $=H^{1}\left(Y, G_{m}\right)=\mathrm{H}^{1}\left(Y, U_{k}\right)$ (cohomology in the Zariski topology).

THEOREM 4. Pic $\left(\left[(n, m), \lambda_{1}, \cdots, \lambda_{n+m}\right] / G_{m}\right)=\mathbf{Z}$.

Proof. Let $U$ be the open cover of $V / G_{m}$ by the $W_{i}$ and let $U^{\prime}$ be the open cover of $V^{\prime} / G_{m}$ by the $W_{i}{ }^{\prime}$. The Cech-to-derived functor cohomology spectral sequences give rise to the following exact sequences of low degree:

$$
\begin{gathered}
1 \rightarrow H^{1}\left(U, U_{k}\right) \rightarrow H^{1}\left(V / G_{m}, U_{k}\right) \rightarrow H^{0}(U, \text { Pic }) \\
1 \rightarrow H^{1}\left(\mathcal{U}^{\prime}, U_{k}\right) \rightarrow H^{1}\left(V^{\prime} / G_{m}, U_{k}\right) \rightarrow H^{0}\left(\mathcal{U}^{\prime}, \text { Pic }\right)
\end{gathered}
$$

We begin by analyzing $(* *)$. $\operatorname{Pic}\left(W_{i}{ }^{\prime}\right)=1$ for each $i$, hence $H^{0}(\mathcal{U}, \mathrm{Pic})=1$ and $H^{1}\left(\mathcal{U}^{\prime}, U_{k}\right)=H^{1}\left(V^{\prime} / G_{m}, U_{k}\right)=\operatorname{Pic}\left(V^{\prime} / G_{m}\right)=\mathbf{Z}$, using proposition 3.

Next we calculate $H^{1}\left(U, U_{k}\right)$. We have the following commutative diagrams:

$$
\begin{aligned}
& (* * *) \\
& \begin{array}{c}
1 \rightarrow B^{1}\left(\mathcal{U}, U_{k}\right) \rightarrow Z^{1}\left(\mathcal{U}, U_{k}\right) \rightarrow H^{1}\left(\mathcal{U}, U_{k}\right) \rightarrow 1 \\
\qquad B^{1}\left(\mathcal{U}^{\prime}, U_{k}\right) \rightarrow Z^{1}\left(\mathcal{U}^{\prime}, U_{k}\right) \rightarrow H^{1}\left(\varkappa^{\prime}, U_{k}\right) \rightarrow 1
\end{array} \\
& 1 \rightarrow Z^{1}\left(\mathcal{U}, U_{k}\right) \rightarrow \prod U_{k}\left(W_{i} \cap W_{j}\right) \rightrightarrows \prod U_{k}\left(W_{i} \cap W_{j} \cap W_{k}\right) \\
& (* * *) \quad \downarrow f \quad \downarrow g \quad \downarrow \\
& 1 \rightarrow \mathrm{Z}^{\mathrm{l}}\left(\mathcal{U}^{\prime}, U_{k}\right) \rightarrow \prod U_{k}\left(W_{i}{ }^{\prime} \cap W_{j}{ }^{\prime}\right) \rightrightarrows \prod U_{k}\left(W_{i}{ }^{\prime} \cap W_{j}{ }^{\prime} \cap W_{k}{ }^{\prime}\right)
\end{aligned}
$$

Since $W_{i}{ }^{\prime}=k^{(n+m-1)}$ and $W_{i}=W_{i}{ }^{\prime} / G, U_{k}\left(W_{i}{ }^{\prime}\right)=U_{k}\left(W_{i}\right)=1$. Thus in $(* * *), B^{1}\left(U, U_{k}\right)=B^{1}\left(U^{\prime}, U_{k}\right)=1$, and hence $Z^{1}\left(U, U_{k}\right)=$ $H^{1}\left(u, U_{k}\right)$ and $Z^{1}\left(U^{\prime}, U_{k}\right)=H^{1}\left(U^{\prime}, U_{k}\right)=Z$. Now $U_{k}\left(W_{i}{ }^{\prime} \cap W_{j}{ }^{\prime}\right)=$ $\mathbf{Z}$, and $U_{k}\left(W_{i} \cap W_{j}\right)$ is a non-trivial subgroup of $U_{k}\left(W_{i}{ }^{\prime} \cap W_{j}{ }^{\prime}\right)$, since $W_{i} \cap W_{j}=\left(W_{i}{ }^{\prime} \cap W_{j}{ }^{\prime}\right) / G$. Similarly, $U_{k}\left(W_{i} \cap W_{j} \cap W_{k}\right)$ is contained in $U_{k}\left(W_{i}{ }^{\prime} \cap W_{j}{ }^{\prime} \cap W_{k}{ }^{\prime}\right)$. Now in the diagram (****), the vertical maps are injections, and the cokernel of $f$ injects into the cokernel of $g$. But the cokernel of $g$ is torsion by the above remarks, and hence the cokernel of $f$ is torsion, hence $Z^{1}\left(U, U_{k}\right)$ is infinite cyclic. Thus $H^{1}\left(u, U_{k}\right)=\mathrm{Z}$.

Now we return to the sequence $(*)$. For each $i, W_{i}$ is affine and $\operatorname{Pic}\left(W_{i}\right)$ is contained in $C \ell\left(W_{i}\right)$. Since $W_{i}=W_{i}{ }^{\prime} / G$ and $C \ell\left(W_{i}{ }^{\prime}\right)=1$, 
by $\left[1,16.1\right.$, p. 82] $C \ell\left(W_{i}\right)$ is contained in the finite group $H^{1}\left(G, k\left[W_{i}{ }^{\prime}\right]^{*}\right)=\operatorname{Hom}\left(G, k^{*}\right)$. Thus $\operatorname{Pic}\left(W_{i}\right)$ is finite, and hence $H^{0}(\mathcal{U}, \mathrm{Pic})$, which is a subgroup of $\prod \mathrm{Pic}\left(W_{i}\right)$, is finite. It follows from (*) that $H^{1}\left(V / G_{m}, U_{k}\right)$ is finitely generated of rank one. To complete the proof, we must show that $H^{1}\left(V / G_{m}, U_{k}\right)=\operatorname{Pic}\left(V / G_{m}\right)$ is torsion free. But this follows from [3, Lemma 4] (again, we note that the reference does not use separation in the proof).

We can now compute the relevant rings of invariants. We first observe that, in the notation of the discussion following Theorem 2, $k\left[V^{\prime}\right]^{G_{m}}=k\left[C^{\prime}\right]$. It follows that $k[V]^{G_{m}}=\left(k\left[V^{\prime}\right]^{G_{m}}\right)^{G}=k\left[C^{\prime}\right]^{G}$ $=k[C]$. Also, $C-C_{0}$ and $C^{\prime}-C_{0}^{\prime}$ are single points. Since $C$ and $C^{\prime}$ have dimension $n+m-1$, if $n>1$ or $m>1, C \ell\left(C_{0}\right)=C \ell(C)$.

Theorem 5. Suppose $n>1$ and $m>1$. Then $k\left[\left[(n, m), \lambda_{1}, \cdots\right.\right.$, $\left.\lambda_{n+m}\right]^{G_{m}}$ has class group $\mathbf{Z}$.

Proof. Let $C_{00}$ be the non-singular locus of $C_{0}$. Since $C_{0}$ is normal, codim $\left(C_{0}-C_{00}\right) \geqq 2$, so $C \ell\left(C_{0}\right)=C \ell\left(C_{00}\right)$, and since $C_{00}$ is nonsingular, $C \ell\left(C_{00}\right)=\operatorname{Pic}\left(C_{00}\right)$. Thus we need to compute $\operatorname{Pic}\left(C_{00}\right)$. Since $n>1$ and $m>1$, codim $\left(V-V_{0}\right) \geqq 2$, hence $U_{k}\left(V_{0}\right)=1$. Let $V_{00}$ be the inverse image of $C_{00}$ in $V_{0}$; since $C_{00}=V_{00} / G_{m}$ and codim $\left(C_{0}-C_{00}\right) \geqq 2$, codim $\left(V_{0}-V_{00}\right) \geqq 2$, so $U_{k}\left(V_{00}\right)=1$. By $[3$, Lemma 4], $\operatorname{Pic}\left(C_{00}\right)$ is torsion free. Let $C_{00}^{\prime}$ be the inverse image of $C_{00}$ in $C_{0}{ }^{\prime}$, so $C_{00}=C_{00}^{\prime} / G$. Let $V_{00}^{\prime}$ be the inverse image of $C_{00}^{\prime}$ in $V_{0}{ }^{\prime}$, so $V_{00}^{\prime} / G_{m}=C_{00}^{\prime}$. As before, $\operatorname{codim}\left(C_{0}{ }^{\prime}-C_{00}^{\prime}\right) \geqq 2$, so $U_{k}\left(V_{00}^{\prime}\right)$ $=1$. Now $V_{00}^{\prime} \rightarrow C_{00}^{\prime}$ is a fibration with fibre $G_{m}$, so, as in Proposition 3 above, [2, Theorem 5] shows that $\operatorname{Pic}\left(C_{00}^{\prime}\right)=Z$. Now we consider the diagram

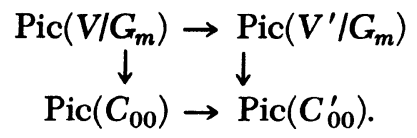

Since $\operatorname{codim}\left(V^{\prime} / G_{m}-C_{00}^{\prime}\right)=\operatorname{codim}\left(V / G_{m}-C_{00}\right) \geqq 2$, the vertical arrows are injections, and in the proof of Theorem 4 we saw that the top horizontal arrow is an injection. Since $C_{00}^{\prime} \rightarrow C_{00}=C_{00}^{\prime} / G$ is finite, the bottom horizontal arrow has torsion kernel. But we know $\operatorname{Pic}\left(C_{00}\right)$ to be torsion free. Since $\operatorname{Pic}\left(V / G_{m}\right)=\mathrm{Z}$ and $\operatorname{Pic}\left(C_{00}^{\prime}\right)=\mathrm{Z}$, it follows that $\operatorname{Pic}\left(C_{00}\right)$ is also infinite cyclic, which completes the proof.

In order to treat the case $n=1$ or $m=1$, we will need the following number-theoretic observation 
LemMA 6. Let $a, b, c$ be relatively prime positive integers. The g.c.d. $(b, c)$ is the smallest positive integer $e$ such that there is a pos tive integer $f$ with $e a+f b \equiv 0(\bmod c)$.

Since the cases $n=1$ and $m=1$ are symmetric, we focus on th case $m=1$.

THEOREM 7. Let $\lambda_{1}, \cdots, \lambda_{n+1}$ be relatively prime. Then $k[[(n, 1), \lambda$ $\left.\left.\cdots, \lambda_{n+1}\right]\right]^{i} G_{m}$ has finite cyclic class group of order $N$, where $N$ defined as follows: let $\mathrm{e}_{\mathrm{i}}=$ g.c.d. (g.c.d. $\left.\left(\lambda_{1}, \cdots, \hat{\lambda}_{i}, \cdots, \lambda_{n}\right), \lambda_{n+1}\right)$ for $i=$ $1,2, \cdots, n$. Then $N=\lambda_{n+1} \mid e_{1} \cdots e_{n}$.

Proof. We first consider the case $\lambda_{n+1}=1$. For $i=1,2, \cdots$, let $t_{i}=x_{i} x_{n+1}^{\lambda_{i}}$, and let $Y^{\prime}=\left[(n, 1), \lambda_{1}, \cdots, \lambda_{n}, 1\right]$. Then it is easy check that $k\left[Y^{\prime}\right]^{G_{m}}=k\left[t_{1}, \cdots, t_{n}\right]$ is a polynomial ring and hence $h$ trivial class group. Now let $Y=\left[(n, 1), \lambda_{1}, \cdots, \lambda_{n+1}\right]$ and let $\psi: Y^{\prime} \rightarrow$ be $\psi\left(x_{1}, \cdots, x_{n+1}\right)=\left(x_{1}, \cdots, x_{n}, x_{n+1}^{\lambda_{n+1}}\right) . \quad H=\Gamma\left(\lambda_{n+1}\right)$ acts on $Y^{\prime} b$ $\beta\left(x_{1}, \cdots, x_{n+1}\right)=\left(x_{1}, \cdots, \beta x_{n+1}\right)$, and $\psi$ induces an isomorphism $Y^{\prime} / H$ with $Y$. Also, $\psi$ is a morphism of spaces with $G_{m}$-action, and th actions of $H$ and $G_{m}$ on $Y^{\prime}$ commute. Let $S=k\left[Y^{\prime}\right]^{G_{m}}$ and let $R$ : $k[Y]^{G_{m}}$. It follows that $S^{H}=R$, so that by $[1,16.1, \mathrm{p}$. 82] there is a exact sequence $1 \rightarrow C l(R) \rightarrow H^{1}\left(H, k^{*}\right) \rightarrow \operatorname{Div}(\operatorname{S})^{H} / \operatorname{Div}(R) \rightarrow 1$; th right hand 1 coming from the fact that $C \ell(S)=1 . H$ is cyclic of ord $\lambda_{n+1}$ and hence so is $H^{1}\left(H, k^{*}\right)$ : if $\alpha$ is a generator of $H, \alpha$ sends $t_{i}$ $\boldsymbol{\alpha}_{t_{\mathrm{i}}}^{\lambda_{1}}$ and since g.c.d. $\left(\lambda_{1}, \cdots, \lambda_{n+1}\right)=1, H$ acts faithfully on $S$.

We need to compute the order of $\operatorname{Div}(S)^{H} / \operatorname{Div}(R)$. Choose positi integers $b_{1}, \cdots, b_{n}$ such that $\sum b_{i} \lambda_{i} \equiv 1\left(\lambda_{n+1}\right)$, and let $u=t_{1}{ }^{1} \cdots t_{\mathrm{n}}$ Then $\alpha(u)=\alpha u$, so $u$ is a primitive element for the quotient field $S$ over the quotient field of $R$, with minimal polynomial $f=T^{d}-u$ where $d=\lambda_{n+1}$. Then $f^{\prime}(u)=d u^{d-1}$. By $[1,16.3$, p. 84], the heig] one primes of $S$ which ramify are among $P_{1}=\left(t_{1}\right), \cdots, P_{n}=\left(t_{n}\right)$. L $e_{i}$ be the ramification index of $P_{i}$. The above exact sequence shows th $C \ell(R)$ is cyclic of order $d / e_{1} \cdots e_{n}$. To complete the proof, we mu show that $e_{i}$ is the desired g.c.d.

Now $R$ is generated over $k$ by invariant monomials, and hence is the least positive integer such that there is an invariant monomial degree $e_{i}$ in $t_{i}$. For convenience, we may assume $i=1$, and let $w$ $t_{1}{ }^{e^{i}} t_{2}{ }^{b_{2}} \cdots t_{n} b_{n}$ be an invariant monomial. Then $e_{1} \lambda_{1}+\sum b_{i} \lambda_{i} \equiv$ $(\bmod d)$, so $e_{1}$ is at least as large as g.c.d. (g.c.d. $\left.\left(\lambda_{2}, \cdots, \lambda_{n}\right), d\right)$. If co versely $e$ is that g.c.d., there are positive integers $c_{2}, \cdots, c_{n}$ such th $e \lambda_{1}+\sum c_{i} \lambda_{i} \equiv 0(d)$, and hence $w^{\prime}=t_{1}{ }^{e} t_{2}{ }^{c_{2}} \cdots t_{n}{ }^{c_{n}}$ is an invaria monomial, so $e_{1} \leqq e$. Thus $e_{1}=e$ and the result follows.

The assumption in Theorem 7 that g.c.d. $\left(\lambda_{1}, \cdots, \lambda_{n+1}\right)=1$ is th 
the action is reduced, and, by the remark following definition 1 , this can always be assumed.

We conclude with an illustration of Theorem 7: $C \ell(k[[(2,1)$, $1,1, n]])=\mathrm{Z} / n \mathrm{Z}$ for any positive $n$ : for g.c.d. $(1, n)=1$. A. Geramita and $M$. Krusemeyer (unpublished) have computed generators and relations of $k[[(2,1), 1,1, n]]^{G_{m}}=R$ : let $S=k\left[s_{0}, \cdots, s_{n}\right]$ map to $R$ by $s_{0} \rightarrow x_{2}{ }^{n} x_{3}, s_{1} \rightarrow x_{1} x_{2}{ }^{n-1} x_{3}, \cdots, s_{n-1} \rightarrow x_{1}{ }^{n-1} x_{2} x_{3}, s_{n} \rightarrow x_{1}{ }^{n} x_{3}$. This is surjective, and the kernel is the ideal generated by the $2 \times 2$ minors of

$$
\left[\begin{array}{c}
s_{0} s_{1} \cdots s_{n-1} \\
s_{1} s_{2} \cdots s_{n}
\end{array}\right] .
$$

\section{REFERENCES}

1. R. Fossum, The Divisor Class Group of a Krull Domain, Springer-Verlag, New York, 1973.

2. A. Magid, The Picard sequence of a fibration, Proc. Amer. Math. Soc. 53 (1975), 37-40.

3. - Fundamental, Class, and Picard groups of rings of invariants, preprint.

4. C. Seshadri, Quotient spaces modulo reductive algebraic groups, Amer. J. Math. 95 (1972), 511-556.

University of Olkahoma, Norman, Oklahoma 73069 
